\title{
Bunker purchasing with contracts
}

\section{Plum, Christian Edinger Munk; Neergaard Jensen, Peter; Pisinger, David}

\section{Published in:}

Maritime Economics \& Logistics

Link to article, DOI:

$10.1057 / \mathrm{mel} .2014 .7$

Publication date:

2014

Document Version

Peer reviewed version

Link back to DTU Orbit

Citation (APA):

Plum, C. E. M., Neergaard Jensen, P., \& Pisinger, D. (2014). Bunker purchasing with contracts. Maritime Economics \& Logistics, 16, 418-435. https://doi.org/10.1057/mel.2014.7

\section{General rights}

Copyright and moral rights for the publications made accessible in the public portal are retained by the authors and/or other copyright owners and it is a condition of accessing publications that users recognise and abide by the legal requirements associated with these rights.

- Users may download and print one copy of any publication from the public portal for the purpose of private study or research.

- You may not further distribute the material or use it for any profit-making activity or commercial gain

- You may freely distribute the URL identifying the publication in the public portal

If you believe that this document breaches copyright please contact us providing details, and we will remove access to the work immediately and investigate your claim. 


\title{
Chapter 7
}

\section{Bunker Purchasing with Contracts}

\author{
Christian E. M. Plum* ${ }^{\ddagger}$ Peter N. Jensen ${ }^{\dagger}$ David Pisinger* \\ *Department of Management Engineering, Technical University of Denmark, \\ Produktionstorvet, Building 426, DK-2800 Kgs. Lyngby, Denmark \\ cemp@man.dtu.dk, pisinger@man.dtu.dk \\ $\ddagger$ Maersk Line \\ Esplanaden 50, DK-1098 København K. \\ mikkel.muhldorff.sigurd@maersk.com \\ $\dagger$ Maersk Oil Trading \\ Esplanaden 50, 1098 København K., Denmark \\ peter.neergaard.jensen@maersk.com
}

\begin{abstract}
1
Abstract The cost for bunker fuel represents a major part of the daily running costs of liner shipping vessels. The vessels, sailing on a fixed roundtrip of ports, can lift bunker at these ports, having differing and fluctuating prices. The stock of bunker on a vessel is subject to a number of operational constraints as capacity limits, reserve requirements and sulphur content. Contracts are often used for bunker purchasing, ensuring supply and often giving a discounted price. A contract can supply any vessel in a period and port, and is thus a shared resource between vessels, which must be distributed optimally to reduce overall costs. The Bunker Purchasing with Contracts Problem (BPCP) has been formulated as a mixed integer program, which has been Dantzig-Wolfe decomposed. To solve it, a novel column generation algorithm has been developed. The algorithm has been run on a series of real world instances with up to 500+ vessels and $500+$ contracts, and provide near optimal solutions. A MIP model cannot solve these instances due to memory requirements.
\end{abstract}

Keywords Bunker purchasing, Liner shipping, Mathematical programming, Maritime optimization, Decomposition methods.

\subsection{Introduction}

Liner shipping companies are at the core of the worlds supply chains providing cheap, compared to any other transport mode, and reliable transport from any corner of the world. This industry has grown massively in the last decades, often with two digit percentage growth rates. But lately the supply of vessels have exceeded the demand for container transport, resulting in many liner

${ }^{1}$ Submitted for Maritime Economics and Logistics 
carriers being loss giving. The profit margins in liner shipping are very slim, with marginal changes resulting in a company loss instead of profit.

This has shifted the shipping industry from a revenue optimizing focus, to use more resources on controlling and minimizing their costs. An example is the spend on bunker fuel, as this constitutes a very large part of the variable operating cost for the vessels.

For liner shipping companies in particular, the purchasing of bunkers can be planned some months ahead, as the vessels are sailing on a fixed schedule allowing for planning, as opposed to other types of shipping. Bunker prices are fluctuating and generally correlated with the crude oil price. But there are significant price difference between ports of up to $100 \$ / \mathrm{mt}$ (of a 600 $\$ / \mathrm{mt}$ price). The price differences between ports are not stable, and the cheapest port on a roundtrip today, may not be the cheapest tomorrow. This creates the need for a frequent (daily) reoptimization of the bunker plan for a vessel, to ensure the lowest bunker costs. An example of a bunkering plan can be seen in Table 7.1 .

Bunker Contracts The market for bunker trading is commoditized and liquid, the use of contracts for a specified amount, port and price (or discount to some price-index) is widespread. This is done to reduce both delivery and price risk and to leverage the strength of being a large player on this market.

Liner shipping companies engage in contracts for the purchase of bunkers at ports where they have a large and regular demand. This is done both to gain a discount compared to the spot market, by leveraging on the large volumes involved, and to increase supply certainty. An example of a liner shipping service can be seen in Figure 7.1. Bunker contracts will usually concern total lifted volumes within a calendar month, with specified minimum and maximal quantities.

The price can be agreed on in different manners, usually by using a fixed discount below the monthly average of a bunker index (Bunkerwire [5]) of the port in question. A contract is for one or more bunker grades and one or more ports, which will be close geographically and considered as the same market. Many contracts can be available in a port for a bunkering vessel, and it must then be chosen which, if any, to purchase bunker from. Spot bunker is assumed freely available at all ports with published prices.

Bunker is available in many different variations, grouped in different grades defined by ISO specifications. Two main parameters for a bunker's quality is its viscosity and its sulphur content. Lower grade (more viscous) bunker is considered better as it places less restrictions on the engine burning the bunker, and lower sulphur content is considered better, because of its lighter environmental footprint. In practice you will always buy the highest viscosity bunker, burnable by your ships engine and available in the port, hence the bunkers viscosity is not considered in this work.

The sulphur content must be considered as an increasing number of regions of the world have $S O_{x}$ Emission Control Areas SECA, for details refer to DNV [10. To model this, two bunker types are considered High Sulphur Fuel Oil, HSFO or $H$ and Low Sulphur Fuel Oil, LSFO or L. The price difference between these varies, but often HSFO is $30 \$ / \mathrm{mt}$ cheaper than LSFO.

The model considered in this paper uses a crystal ball approach, i.e. using data not known at decision time, to benchmark the quality of already executed decisions. As the actual price of the contract is not known before a month has passed, the model will use after-the-fact prices for calculations.

The problem is to satisfy the vessels consumption by purchasing bunkers at the minimum overall cost, while considering reserve requirements, other operational constraints and adhering to a number of bunker contracts, the Bunker Purchasing with Contracts Problem. A novel decomposition method is presented for BPCP, and the first results, solving BPCP to near optimality are presented for large real world instances.

\subsubsection{Literature}

In this section we relate to literature relevant for this study within maritime optimization, liner shipping and bunker usage and purchasing in particular. For a broad introduction to shipping and the importance of bunker spend refer to Stopford [20] and for an introduction to operations 
research within the maritime industry Christiansen et al. [7] and Christiansen et al. 8] provides excellent overviews. A detailed description of Liner Shipping Network Design and, the impact of bunker usage and other relevant factors appears in Brouer et al. 4. They also introduce LINERLIB 2012, a benchmark data suite, consisting of liner shipping relevant data and benchmarks specifically for liner shipping network design problems. Details on the bunkering industry in relation to shipping can be found in Boutsikas [3].

For a vessel sailing on a given port to port voyage at a given speed, the bunker consumption can be fairly accurately predicted. This gives an advantage in bunker purchasing, when a vessel has a stable schedule known for some months ahead. The regularity in the vessel schedules in liner shipping allows for detailed planning of a specific vessel, as considered in the works of Plum and Jensen [18, Besbes and Savin [2] and Yao et al. 23]. These papers consider variants of a bunker optimization problem considering a single vessel. The work of Plum and Jensen [18 considers multiple tanks in the vessel and stochasticity of both prices and consumption. Yao et al. 23. does not consider stochastic elements nor tanks, but has vessel speed as an variable of the model. Bunker contracts are not considered in these.

Besbes and Savin 2] consider different refueling policies for liner vessels and has some good considerations on the modeling of stochastic bunker prices using markov processes. This is used to show that the bunkering problem in liner shipping can be seen as a stochastic capacitated inventory management problem. Bunker contracts are not considered, neither are other operational constraints than capacity.

The work of Farina [12] is an extension of Plum and Jensen [18] with the additional consideration of bunker contracts, where a MIP model is presented capable of solving a 50 vessel instance for a 6 month period, falling short of solving real world instances of hundreds of vessels. An outline of a similar model and decomposition of this was presented at the ICCL 2012 conference, Farina et al. 13, but without computational results. The effect of the bunker price on Liner Shipping Network Design has been studied in a number of recent papers as Wang and Meng 21] and Meng et al. 16.

The effect of bunker usage by the maritime industry in relation to the bunker price is investigated by Corbett et al. [9] with the aim of reducing $\mathrm{CO}_{2}$ emissions by imposing tax on bunkers. The work of Acosta et al. [1] considers factors impacting the choice of bunker port. Fagerholt et al. 11] considers the optimal speed and route for a ship with respect to bunker costs. Other work on bunker costs and its impact on maritime transportation includes Notteboom and Vernimmen [17, who consider how slow steaming and the cost structure of liner shipping networks are affected by changes in bunker costs, and Ronen [19, who considers the bunker price's effect on speed and fleet size. The recent work of Wang et al. 22] provides an overview of available bunker optimization methods in shipping.

Contribution The contribution of this paper is a model considering contracts and other operational constraints, as reserve requirements and minimal lift quantities, relevant when purchasing bunker for a liner shipping company. This model has been Dantzig-Wolfe decomposed and a novel column generation algorithm created. It is discussed, how the decomposition allows for the dual values of the contracts to be investigated. Finally, an implementation of the algorithm shows it can solve very large problems, met in real world problem instances. As stated in the Summary and Future Work of Besbes and Savin [2]:

The single-vessel approach developed in this paper can serve as a good initial step to optimizing fleet profits. At the same time, real business settings are often characterized by volume refueling discounts, which can only be fully exploited using more than one vessel. Thus, the development of multiple-vessel profit management models represent a challenging research direction that will be of immediate interest to practitioners.

The structure of the paper is as follows. Section 7.2 explains the mathematical notation used throughout the paper. A Mixed Integer Programming model for BPCP is formulated in Section 
7.2.1. The model is Dantzig-Wolfe (DW) decomposed and Column Generation algorithm is devised in Section 7.3 Computational results and comparison of the MIP and the DW algorithm is presented in Section 7.4, where it can be seen that DW can solve many instances, which can not be solved by the MIP model. Conclusion and outlook is given in section 7.5 .

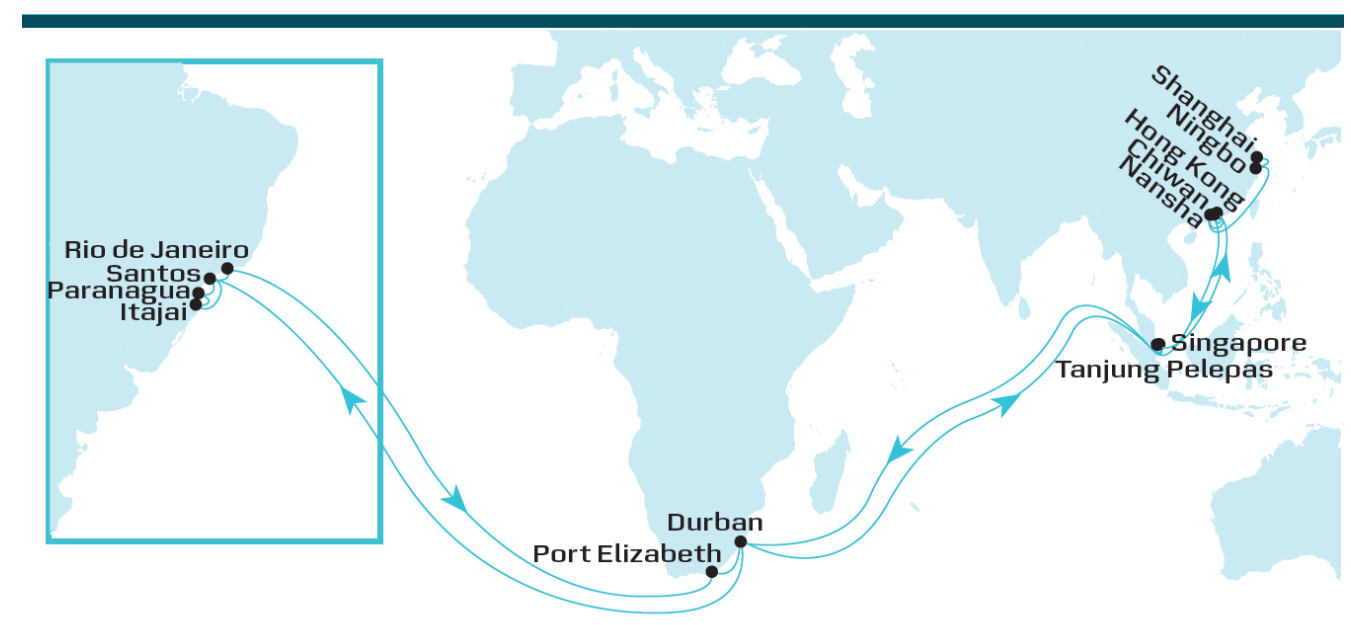

Figure 7.1: The ASAS 2 Service, transporting containers between East Coast South America, South Africa and the Far East. This service allows for bunkering in three distinct markets.

\subsection{Bunker Purchasing with Contracts}

We introduce the mathematical notation used throughout the paper. Let $v \in V$ be the set of vessels. Let $i \in I$ be an ordered set of port calls, the vessel's schedule. A port call $i$ will be uniquely defined by a port, a vessel, $v(i)$ and a date. Let $\operatorname{init}(v)$ and $\operatorname{term}(v)$ be the first and last considered port call of vessel $v$. Let $b \in B=\{L, H\}$ be the two considered bunker types. The startup cost for bunkering at a port call $i$, is startcost $t_{i}$. Each vessel, $v$ has a capacity $D_{v, b}$ for each bunker type, $b$. For each leg $i$ of the schedule, the vessel consumes $F_{i, b}$ bunker, between port call $i$ and $i+1$.

Contract bunker must be purchased according to details given by a number of contracts $c \in$ $C$, minimal and maximal quantities are given by $\underline{q}_{c}$ and $\bar{q}_{c}$. The specified quantities are soft constraints, which can be violated by paying a high cost, $\underline{w}$, for violating the minimum volume and a lower cost for breaking the maximal constraint, $\bar{w}$. Contract $c$ may cover several ports and multiple vessels can call at these ports in the duration of the contract. Each contract will give rise to a number of purchase options, $m \in M$, i.e. discrete events where a specific port call $i$, and thus vessel $v$, calls within a time period, allowing it to purchase bunker from a contract $c$. Purchases on a purchase option $m$ will be done at a price $p_{m}$, specified by the contract $c$. To simplify modelling and to increase the density of the derived model, the sets of port calls, $i \in I$ and purchase options, $m \in M$ will be used instead of their underlying sets: ports, vessels and contracts, which could give an equivalent but much larger model.

The possibility of purchasing on the spot market, is considered as a special type of contract. The minimal and maximal volumes are relaxed as $\underline{q}_{c}=0$ and $\bar{q}_{c}=\infty$. All port calls $i$ have two spot purchase options $m$ for LSFO and HSFO, with prices set at the corresponding spot price of the day and port. For ports where bunker prices are not published, we assume a high cost.

The variables of the model are: $l_{m}$ the purchase of bunker for each purchase option $m$. The binary variable $\delta_{i, b}$ is set iff a purchase of a bunker type $b$ is made at a port call $i$. The volume 
of bunker after a vessel leaves port, $h_{i, b}$ is a continuous variable, as is the consumption of each bunker type on vessel between port $i$ and $i+1, f_{i, b}$. The contract violation or slack variables are $\underline{s}_{c}$ and $\bar{s}_{c}$.

\subsubsection{Model}

The BPCP can be formulated as a Mixed Integer Program:

$$
\min \sum_{i \in I} \sum_{b \in B}\left(\delta_{i, b} \cdot \text { startcost }_{i}\right)+\sum_{m \in M}\left(p_{m} \cdot l_{m}\right)+\sum_{c \in C}\left(\underline{s}_{c} \cdot \underline{w}+\bar{s}_{c} \cdot \bar{w}\right)
$$

Subject to

$$
\begin{array}{rc}
h_{i, b}=h_{i-1, b}+\sum_{m \in M(i, b)} l_{m}-f_{i-1, b} & \forall i, b \\
f_{i, b} \leq h_{i, b} & \forall i, b \\
\sum_{b \in B} f_{i, b}=F_{i, H}+F_{i, L} & \forall i \\
f_{i, L} \geq F_{i, L} & \forall i \\
h_{i, b} \leq D_{v(i), b} & \forall i, b \\
\underline{q}_{c}-\underline{s}_{c} \leq \sum_{m \in M(c)} l_{m} \leq \bar{q}_{c}+\bar{s}_{c} & \forall c \\
\sum_{m \in M(i, b)} l_{m} \leq \delta_{i, b} \cdot D_{v, b} & \forall i, b
\end{array}
$$

The objective minimizes startup costs, bunker cost and contract violation penalties. The constraints (7.1) ensures flow conservation at each port, vessel and bunker type. Constraints 7.2 ensures that no more bunker than available is used between port $i$ and $i+1$. Constraints (7.3) and (7.4) maintains the consumption of bunker, allowing LSFO to substitute HSFO, but not opposite. The bunker capacity of the vessels are enforced by constraints 7.5 . The minimal and maximal quantity required by the contracts are ensured by double sided constraints (7.6), allowing for violations. The decision variables $\delta_{i, b}$ are set by constraints (7.7).

To facilitate use in a benchmark setting, initialization and termination criteria for start and end bunker volumes must also be set:

$$
\begin{array}{rc}
h_{i(0, v), b}=h_{b}^{\text {init }(v)} & \forall v, b \\
\sum_{b \in B} h_{i(t, v), b} \geq \sum_{b \in B} h_{b}^{t e r m}(v) & \forall v \\
h_{i(t, v), L} \geq h_{L}^{t e r m}(v) & \forall v
\end{array}
$$

Variable domains:

$$
\begin{aligned}
h_{i, b}, l_{m}, f_{i, b}, \underline{s}_{c}, \bar{s}_{c} \in \mathbb{R}^{+} & \forall i, b, m, c \\
\delta_{i, b} \in\{0,1\} & \forall i, b
\end{aligned}
$$

\subsubsection{Operational Constraints}

In practice bunker purchasing in liner shipping is influenced by a wide range of operational, commercial and financial factors, which dictates the properties of a good bunker plan. Some of these are described here and a few are formulated as constraints, refer to earlier mentioned literature for an elaborate discussion of other factors. 
As the consumption of bunker on a leg is an uncertain parameter due to factors as changed schedule (and thus speed), wind, current, waves and hull roughness, a good bunker plan will allow for variation in the bunker consumption. A way to handle this is to enforce a minimum reserve requirement of bunker at port arrival. This can be modeled as in 7.13 , where $F_{i}$ is the minimal reserve requirement at port arrival.

Besides the startup cost for bunkering, startcost ${ }_{i}$, bunker suppliers will usually require a minimum quantity to be purchased at each bunkering, this can be handled with constraints (7.14), where $\underline{L}_{i, b}$ is the minimal quantity.

$$
\begin{aligned}
F_{i} \leq \sum_{b \in B}\left(h_{i, b}-\sum_{m \in M} l_{m}\right) & \forall i \\
& \delta_{i, b} \cdot \underline{L}_{i, b} \leq \sum_{m \in M} l_{m} \quad \forall i, b
\end{aligned}
$$

Capital and carriage cost The capital costs of bunker is extensive, due to the large volumes and high prices. A model could consider this by adding this cost (or lacking interest) to the objective, proportional to the average load of bunker on the vessels. Similarly a vessel carrying a large volume of bunker will, all things equal, have a larger draft. This will in general (but not always, due to specifics in the vessels design as the bulb) imply an increased bunker consumption proportional to an increased load. With realistic values of this relation, this term could be considered in the objective in the same manner as the capital costs.

California sales tax The California bunker sales tax, as described by California Legislative Analyst's Office [6], imposes a tax on bunker bought in California, which necessarily must be used enjourney to the first out of state port. I.e. if a vessel arrives with $1000 \mathrm{mt}$ at an Californian port and requires $2000 \mathrm{mt}$ to reach the first non-californian port on its schedule, it must pay a tax for the first $1000 \mathrm{mt}$ purchased. With additional decision variables this can be modelled and included in the objective.

Contract min/max volumes \& port call max volumes Contracts may have minimum and maximal volumes that must be lifted per purchase. This can be modelled similarly to the minimum lift constraints. As can spot purchases at port calls have maximal lift restrictions due to short port stays or limited supply.

Quarantine A sample is usually taken from purchased bunker, to be analyzed for its specific content of carbohydrates, sulphur, water, ashes, etc. the ample must be within the ISO specifications of the purchased bunker grade. Until the result of the laboratory test are received, the bunker may not be used. This test can take 3-5 days. This constraint can be considered by increasing the reserve requirements at port calls with bunker purchased within the last 5 days.

Constraints for capital and carriage cost, California sales tax, contract $\mathrm{min} / \mathrm{max}$ volumes, port call max volumes and quarantine have not been implemented, to limit the required implementation effort. All of them can be formulated linearly and only relate to a single vessel at a time, allowing them to be considered in a vessel specific subproblem.

\subsubsection{Complexity}

The problem is weakly NP-hard by reduction from the knapsack problem, as described in Kellerer et al. 14. From the knapsack problem in minimization form: Given a set $N$ of items having profit $p_{i}$ and weight $w_{i}$ and a knapsack of capacity $c$, the problem is to fill the knapsack at minimum overall profit, such that the overall weight is at least $c$. Given an instance of the knapsack problem, we construct an instance of the bunker purchasing problem by having one vessel, visiting $N$ ports. The fuel consumption between each pair of ports is 0 , except the leg after the last port visit, where 
the consumption is $c$. In each port, we have a contract of maximum $w_{i}$, and the minimum limit for lifting bunker is also $w_{i}$. The cost of buying the quantity $w_{i}$ is $p_{i}$. It is easily seen that solving the bunker purchasing problem also solves the knapsack problem.

\subsection{Algorithm}

The fleet of a global liner shipping company may consist of hundreds of vessels, with many of these having overlapping schedules visiting the same hub ports. This means that the full problem can be of a very large size, making the MIP model impossible to solve for large instances as seen in Section (7.4. This makes it interesting to consider a decomposition of the MIP model, to solve these large problem instances.

The arc flow model given by (7.1) - 7.14 is Dantzig-Wolfe decomposed on the variables $l_{m}$. Let $R_{v}$ be the set of all feasible bunkering patterns for a vessel $v$, satisfying constraints $(7.1)-(7.14)$, except 7.6. This set has an exponential number of elements. Each pattern $r \in R_{v}$ is denoted as a set of bunkerings. Let $u_{r}=\sum_{m \in M}\left(p_{m} \cdot l_{m}\right)+\sum_{i \in I} \sum_{v \in V} \sum_{b \in B}\left(\delta_{i, b} \cdot\right.$ startcost $\left._{i}\right)$ be the cost for pattern $r \in R_{v}$. Let $\lambda_{r}$ be a binary variable, set to 1 iff the bunkering pattern $r$ is used. Let $o_{r, c}$ be the quantity purchased of contract $c$ by pattern $r$. The BPCP can then be formulated as:

$$
\min \sum_{v \in V} \sum_{r \in R_{v}} \lambda_{r} \cdot u_{r}+\sum_{c \in C}\left(\underline{s}_{c} \cdot \underline{w}+\bar{s}_{c} \cdot \bar{w}\right)
$$

Subject to

$$
\begin{array}{cc}
\underline{q}_{c}-\underline{s}_{c} \leq \sum_{v \in V} \sum_{r \in R_{v}} \lambda_{r} \cdot o_{r, c} \leq \bar{q}_{c}+\bar{s}_{c} & \forall c \\
\sum_{r \in R_{v}} \lambda_{r}=1 & \forall v \\
\lambda_{r} \in\{0,1\} & \forall r
\end{array}
$$

The objective minimizes the costs of purchased bunker, startup costs and slack costs. Constraints (7.16) ensures that all contracts are fulfilled. Convexity constraints 7.17) ensure that exactly one bunker pattern is chosen for each vessel.

\subsubsection{Pricing Problem}

Let $\underline{\pi_{c}} \leq 0$ and $\overline{\pi_{c}} \leq 0$ be the dual variables for the upper and lower contract constraints 77.16 , due to the structure of these constraints at least one of these will be 0 for each contract $c$. Let $\theta_{v} \in \mathbb{R}$ be dual variables for the convexity constraints 7.17 . Then the pricing problem becomes:

$$
\text { Min: } u_{r}+\sum_{c \in C}\left(\underline{\pi_{c}}-\overline{\pi_{c}}\right)-\theta_{v}
$$

Subject to constraints 7.1) - 7.14, except 7.6.

This pricing problem is a Mixed Integer Program, considering a single vessel. This size of problem can be solved in reasonable time by a standard MIP solver, as done in Plum and Jensen [18. Columns $\lambda_{r}$ with negative reduced cost will then be added to the master problem, also solved as a MIP.

\subsubsection{Column Generation Algorithm}

Due to the large number of columns the problem is solved by a Column Generation algorithm, where the root node is solved to LP optimality. The root node is then solved with integral property on all columns by a MIP solver. 
Initially all dual variables are set to zero, a subproblem is constructed for each vessel and solved as a MIP problem. The first master problem is then constructed with one solution for each vessel as columns. This master is solved and the first dual values are found. The subproblems are resolved for all vessels (only the objective coefficients for the contracts needs updating) and new columns are generated for the master. This continues until no negative reduced cost columns can be generated, and the LP optimal solution is achieved.

Following, the problem is solved as a MIP, providing an integral solution. The subproblems only need to find a negative reduced costs column, to ensure progress of the algorithm. This means that initially they are allowed to return solutions with considerable subproblem gaps. As the algorithm progresses, the allowable subproblem gap is reduced, until it reaches the tolerance level.

\subsubsection{Dual stabilization}

A simple form of dual stabilization has been used in the implementation to speed up convergence. The Boxstep method described in Marsten et al. [15] imposes a box around the dual variables, which are limited from changing more than $\pi_{\max }$ per iteration. This has been motivated by the dual variables only taking on values $\{-\underline{w}, \bar{w}, 0\}$ in the first iteration, these then stabilize at smaller numerical values in subsequent iterations.

\subsubsection{Interpretation of dual values}

Besides using the developed method for benchmarking the historical performance of bunker purchases, it can be used in a context of evaluating the gain of a considered contract.

Using best estimates for bunker consumption and prices (current prices for instance) together with known or expected contracts a baseline bunker purchasing plan could be run. A new scenario could then be constructed with the addition of the considered contract and by analyzing the output, it could be seen whether the overall costs of the scenario increased or decreased as compared with the baseline.

Another investigation could be to solely consider the baseline's final dual variables, $\pi_{c}$ and $\overline{\pi_{c}}$, and depending on the magnitude of these evaluate the contracts effect. As these dual values are the same for all subproblems, they can be interpreted as balancing out the price of the contract, increasing the price if it is a popular contract or decreasing it otherwise, converging when they are in balance. The magnitude of this will be proportional to the contracts gain.

\subsection{Computational Results}

The proposed algorithm has been implemented in ILOG OPL as modelling language and CPLEX 12.2 as LP/MIP solver, this implementation is referred to as DW. To evaluate the performance of DW the MIP model of Section 7.2.1 has been implemented in CPLEX 12.2.

Real life data for a large number of liner vessels describing their schedules, consumptions, tank capacities and other relevant data has been made available by Maersk Oil Trading, who have also supplied data on a large number of actual bunker contracts and spot prices available in a range of ports. Based on these data a number of instances have been constructed to test the scalability and performance of the implementations. Due to confidentiality reasons the price's have been distorted by $\pm 10 \%$, in order to maintain the structure of the problem. The penalty $\underline{w}$ for violating minimum volume is set at $200 \$ / \mathrm{mt}$, and the penalty $\bar{w}$ for breaking the maximal constraint at $50 \$ / \mathrm{mt}$. If a bunker price is not available at a port, the price is set at $1000 \$ / \mathrm{mt}$. Details about the instances can be seen in Table 7.2 .

\subsubsection{Parameter Tuning}

Three parameters have been tested to improve the running time and performance of the DW algorithm, all test have been performed on 4 medium and large problem instances and the average 
changes in running time and objective value, as compared with the best are shown.

Table 7.5 shows the effect of different values of the maximal Box Step size of the Boxstep method. A value of 1000 indicates that the duals are free, as these are bounded by $\{-\underline{w}, \bar{w}\}$. It can be seen, that the algorithm performs better in terms of time and quality using the boxstep method. A value of 75 is chosen for further runs, as it gives the best tradeoff of running time and objective value.

As described in section 7.3 .2 the subproblems are initially not solved to optimality. Table 7.6 investigates the initial gap the subproblems terminates with. The improvement effect is small, but a benefit arises with an initial gap of 0.01 . Table 7.7 investigates by which factor this gap should decrease until reaching $10 \mathrm{e}-6$. A value of 25 is chosen.

An overview of the performance and results can be found in Tables 7.3 and 7.4 . It can be seen that the DW model is able to solve the problem for all instances. For larger instances MIP runs out of memory and finds no solution, due to the size of the instances and their resulting MIPs. Both models find solutions with very small gaps, but still considerable absolute gap's to the optimal solution. MIP only finds optimal solutions for the smallest instances, for all medium and large instances the solver runs out of memory before it has closed the gap. DW is able to find solutions with relatively small gaps for even the largest problem instances covering all vessels and all contracts on a global level. In practice the resulting gaps of the algorithms, can be much less in reality as they are based on a lower bound.

\subsection{Conclusion and Further Work}

We have presented a MIP model for the Bunker Purchasing with Contracts Problem. This model has been Dantzig-Wolfe decomposed and a novel Column Generation algorithm was presented. The MIP model and the DW algorithm have been implemented and run on very large instances. The DW algorithm is able to find good solutions for all instances within the timelimit, where the MIP model is unable to find solutions before running out of memory. The advantage of the DW algorithm is that many of the constraints can be dealt with in the pricing problem. Additionally the dual information is provided which can be used to evaluate a contract. It has been certified by Maersk Oil Trading that the produced bunker plans are operationally feasible and that contracts are considered by the model in a adequate manner. Maersk Oil Trading will investigate the method in depth, with the aim of implementing its results in their operations, to better utilize their bunker contracts. The algorithm could also be used to evaluate the potential of a new contract, or what the right upper and lower volumes of a contracts should be, in a negotiating setting.

Numerous additional operational constraints can be included in the modeling, as reserve requirements, multiple bunker tanks, mixing penalties and others mentioned in related literature for single vessel bunkering problems. These could be included in the subproblem as they all deal with a single vessel. Further work could be done on the DW to close the optimality gaps, by branching on fractional variables of the root node, devising a Branch-and-Price algorithm.

\section{Acknowledgements}

The authors gratefully acknowledge the support from The Danish Council for Strategic Research to the ENERPLAN project. This work would not have been possible without the adept guidance and support from experienced Maersk Oil Trading and Maersk Line staff, in particular we would like to thank Claus Anker and Mikkel M. Sigurd who have given invaluable support for this project. We also thank Stephen Hall for good support with OPL/CPLEX and Federico Farina for considerable assistance in preparing the data for the investigated instance. 


\section{Bibliography}

[1] Manuel Acosta, Daniel Coronado, and Ma Del Mar Cerban. Bunkering competition and competitiveness at the ports of the gibraltar strait. Journal of Transport Geography, 19(4): $911-916,2011$.

[2] Omar Besbes and Sergei Savin. Going bunkers: The joint route selection and refueling problem. Manufacturing and Service Operations Management, 11(4):694-711, 2009.

[3] Angelos Boutsikas. The bunkering industry and its effect on shipping tanker operations. Master's thesis, Massachusetts Institute of Technology. Dept. of Ocean Engineering., 2004.

[4] Berit D. Brouer, J. Fernando Alvarez, Christian E. M. Plum, David Pisinger, and Mikkel M. Sigurd. A base integer programming model and benchmark suite for liner shipping network design. Transportation Science, 2013. Accepted by Transportation Science.

[5] Platts Bunkerwire. Platts bunkerwire. http://www.platts.com/IM.Platts.Content/ProductsServices/Products/b 2013.

[6] California Legislative Analyst's Office. California bunker sales tax. http://www.lao.ca.gov/2001/bunker_fuel/012501_bunker_fuel.html.

[7] Marielle Christiansen, Kjetil Fagerholt, and David Ronen. Ship routing and scheduling: status and perspectives. Transportation Science, 38(1):1-18, 2004.

[8] Marielle Christiansen, David Ronen, Bo Nygreen, and Kjetil Fagerholt. Maritime transportation. In Handbooks in Operations Research and Management Sciences, pages 189-284. North Holland, 14 edition, 2007.

[9] James J. Corbett, Haifeng Wang, and James J. Winebrake. The effectiveness and costs of speed reductions on emissions from international shipping. Transportation Research Part D: Transport and Environment, 14(8):593 - 598, 2009.

[10] Det Norske Veritas DNV. Sulphur emission control area. http://www.dnv.com.ar/binaries/flyer_marpol_seca_tcm158-278353.pdf.

[11] Kjetil Fagerholt, Gilbert Laporte, and Inge Norstad. Reducing fuel emissions by optimizing speed on shipping routes. The Journal of the Operational Research Society, 61(3):523-529, 2009.

[12] Federico Farina. Bunker optimization with contracts. Master's thesis, Universita Di Bologna, 2012.

[13] Federico Farina, Peter N. Jensen, Christian E. M. Plum, and David Pisinger. Bunker purchasing with contracts. 2012. Presented at http://iwi.econ.uni-hamburg.de/iccl2012/index.htm.

[14] Hans Kellerer, Ulrich Pferschy, and David Pisinger. Knapsack Problems. Springer, 2004.

[15] Roy E. Marsten, William W. Hogan, and Jacob W. Blankenship. The boxstep method for large-scale optimization. Operations Research, 23(3):389-405, May/June 1975.

[16] Qiang Meng, Shuaian Wang, and Zhiyuan Liu. Network design for shipping service of largescale intermodal liners. Transportation Research Record: Journal of the Transportation Research Board, 2269:42-50, 2012.

[17] Theo E. Notteboom and Bert Vernimmen. The effect of high fuel costs on liner service configuration in container shipping. Journal of Transport Geography, 17(5):325-337, 2009.

[18] Christian E. M. Plum and Peter Neergaard Jensen. Minimization of bunker costs. Master's thesis, 2007. 
[19] David Ronen. The effect of oil price on containership speed and fleet size. J Oper Res Soc, 62:211-216, 2010.

[20] Martin Stopford. Maritime Economics. Routledge, third edition, 2009.

[21] Shuaian Wang and Qiang Meng. Robust schedule design for liner shipping services. Transportation Research Part E: Logistics and Transportation Review, 48(6):1093 - 1106, 2012.

[22] Shuaian Wang, Qiang Meng, and Zhiyuan Liu. Bunker consumption optimization methods in shipping: A critical review and extensions. Transportation Research Part E: Logistics and Transportation Review, 53(0):49 - 62, 2013.

[23] Zhishuang Yao, Szu Hui Ng, and Loo Hay Lee. A study on bunker fuel management for the shipping liner services. Computers and Operations Research, 39(5):1160 - 1172, 2012. 


\begin{tabular}{|l|c|c|c|c|c|c|c|c|}
\hline Port Id & \multicolumn{2}{|c|}{ Departure stock $(\mathrm{mt})$} & \multicolumn{2}{c|}{ Consumption $(\mathrm{mt})$} & \multicolumn{2}{c|}{ Purchase $(\mathrm{mt})$} & \multicolumn{2}{c|}{ Spot Price (\$mt) } \\
\hline & LSFO & HSFO & LSFO & HSFO & LSFO & HSFO & LSFO & HSFO \\
\hline NLROT & 462 & 648 & 5 & 0 & 0 & 0 & 481 & 455 \\
DEBRV & 457 & 648 & 100 & 0 & 0 & 0 & 500 & 490 \\
GBFXS & 357 & 648 & 97 & 648 & 0 & 0 & 1000 & 675 \\
USNWK & 260 & 1004 & 0 & 134 & 0 & $186+818$ & 491 & 465 \\
USCHS & 260 & 870 & 0 & 425 & 0 & 0 & 490 & 477 \\
USSAV & 260 & 445 & 0 & 74 & 0 & 0 & 493 & 457 \\
USMIA & 260 & 371 & 0 & 211 & 0 & 0 & 1000 & 1000 \\
USHOU & 260 & 1456 & 0 & 122 & 0 & 1296 & 1000 & 442 \\
USMOB & 260 & 1334 & 183 & 201 & 0 & 0 & 1000 & 1000 \\
USNFK & 77 & 1133 & 24 & 555 & 0 & 0 & 484 & 469 \\
GBFXS & 53 & 578 & 8 & 0 & 0 & 0 & 1000 & 641 \\
NLROT & 1053 & 4314 & 0 & 2340 & 1008 & 3737 & 442 & 421 \\
DEBRV & 1053 & 1974 & 0 & 447 & 0 & 0 & 457 & 447 \\
USNWK & 1053 & 1527 & 2 & 110 & 0 & 0 & 490 & 466 \\
USCHS & 1051 & 1417 & 0 & 25 & 0 & 0 & 495 & 482 \\
USSAV & 1051 & 1392 & 0 & 82 & 0 & 0 & 502 & 471 \\
USMIA & 1051 & 1310 & 0 & 211 & 0 & 0 & 1000 & 1000 \\
USHOU & 1051 & 1099 & 0 & 128 & 0 & 0 & 1000 & 451 \\
USMOB & 1051 & 971 & 0 & 365 & 0 & 0 & 1000 & 1000 \\
USNFK & 1051 & 606 & 221 & 606 & 0 & 0 & 510 & 495 \\
NLROT & 830 & 4021 & 21 & 0 & 0 & 4021 & 436 & 415 \\
GBFXS & 809 & 4021 & 65 & 0 & 0 & 0 & 1000 & 652 \\
DEBRV & 744 & 4021 & 98 & 511 & 0 & 0 & 467 & 456 \\
USNWK & 646 & 3510 & 0 & 161 & 0 & 0 & 485 & 464 \\
USCHS & 646 & 3349 & 0 & 19 & 0 & 0 & 496 & 483 \\
USSAV & 646 & 3330 & 0 & 98 & 0 & 0 & 499 & 468 \\
USMIA & 646 & 3232 & 0 & 183 & 0 & 0 & 1000 & 1000 \\
USHOU & 646 & 3049 & 0 & 135 & 0 & 0 & 1000 & 465 \\
USMOB & 646 & 2914 & 0 & 388 & 0 & 0 & 1000 & 1000 \\
\hline
\end{tabular}

Table 7.1: An example of a bunker plan. Departurestock is the stock of bunker at departure of the port, as calculated by the model. Consumption is, a model input, the consumption of bunker from this port to the next. Purchase is the quantity of bunker purchased at the port and SpotPrice is the market price of bunker at the spot market. Possible bunker contracts are not shown. At the fourth port call 186 $\mathrm{mt}$ HSFO is bought at the spot market and $818 \mathrm{mt}$ HSFO through a contract. 


\begin{tabular}{|l|c|c|c|c|}
\hline Instance & Size & $V$ & $P$ & $C$ \\
\hline RULED & Small & 6 & 1048 & 29 \\
FRFSM & Small & 8 & 2128 & 10 \\
ZADUR & Small & 49 & 5973 & 35 \\
US_WC & Small & 32 & 6022 & 68 \\
USNWK & Medium & 49 & 9048 & 69 \\
USSAV & Medium & 50 & 9194 & 23 \\
PABLB & Medium & 65 & 9817 & 27 \\
AEJAL & Medium & 80 & 15442 & 9 \\
09_H2 & Large & 408 & 16214 & 307 \\
11_H2 & Large & 572 & 18426 & 254 \\
10_H1 & Large & 469 & 18704 & 332 \\
10_H2 & Large & 534 & 21907 & 424 \\
11_H1 & Large & 609 & 23453 & 376 \\
HKHKG & Large & 158 & 29177 & 20 \\
10_FY & Large & 535 & 40611 & 756 \\
\hline
\end{tabular}

Table 7.2: Instances of varying sizes for the BPCP. Instance is the name, Size is a grouping of the instances. $V$ the number of vessels, $P$ the number of port calls, $C$ the number of Contracts.

\begin{tabular}{|l|c|c|c|c|}
\hline Instance & $O b j_{M I P}$ & $L B_{M I P}$ & Gap $_{M I P}$ & $t_{M I P}$ \\
\hline RULED & $5.404 \mathrm{e}+7$ & $5.404 \mathrm{e}+7$ & $0.00 \%$ & 1083 \\
FRFSM & $1.319 \mathrm{e}+8$ & $1.319 \mathrm{e}+8$ & $0.00 \%$ & 21 \\
ZADUR & $7.064 \mathrm{e}+8$ & $7.063 \mathrm{e}+8$ & $0.02 \%$ & 609 \\
US_WC & $6.628 \mathrm{e}+8$ & $6.626 \mathrm{e}+8$ & $0.03 \%$ & 481 \\
USNWK & $9.067 \mathrm{e}+8$ & $9.063 \mathrm{e}+8$ & $0.03 \%$ & 834 \\
USSAV & $9,830 \mathrm{e}+8$ & $9.826 \mathrm{e}+8$ & $0.04 \%$ & 775 \\
PABLB & $1.108 \mathrm{e}+9$ & $1.107 \mathrm{e}+9$ & $0.06 \%$ & 906 \\
AEJAL & $1.490 \mathrm{e}+9$ & $1.489 \mathrm{e}+9$ & $0.03 \%$ & 686 \\
09_H2 & $2.115 \mathrm{e}+9$ & $2.113 \mathrm{e}+9$ & $0.10 \%$ & 1160 \\
11_H2 & $2.478 \mathrm{e}+9$ & $2.475 \mathrm{e}+9$ & $0.09 \%$ & 1107 \\
10_H1 & $2.255 \mathrm{e}+9$ & $2.253 \mathrm{e}+9$ & $0.09 \%$ & 1181 \\
10_H2 & Out of Mem & & & \\
11_H1 & Out of Mem & & & \\
HKHKG & Out of Mem & & & \\
10_FY & Out of Mem & & & \\
\hline
\end{tabular}

Table 7.3: Results and performance of MIP implementation. Instance is the name. $O b j_{M I P}$ is the best found solution for algorithm, $L B_{M I P}$ is the best found lower bound. Gap ${ }_{M I P}$ is the resulting gap between upper and lower bound and $t_{M I P}$ is the timed used in seconds. 


\begin{tabular}{|l|c|c|c|c|}
\hline Instance & $O b j_{D W}$ & $L B_{D W}$ & Gap $_{D W}$ & $t_{D W}$ \\
\hline RULED & $5.408 \mathrm{e}+7$ & $5.404 \mathrm{e}+7$ & $0.08 \%$ & 118 \\
FRFSM & $1.321 \mathrm{e}+8$ & $1.319 \mathrm{e}+8$ & $0.20 \%$ & 86 \\
ZADUR & $7.071 \mathrm{e}+8$ & $7.064 \mathrm{e}+8$ & $0.10 \%$ & 653 \\
US_WC & $6.654 \mathrm{e}+8$ & $6.627 \mathrm{e}+8$ & $0.41 \%$ & 1142 \\
USNWK & $9.077 \mathrm{e}+8$ & $9.066 \mathrm{e}+8$ & $0.11 \%$ & 1114 \\
USSAV & $9.830 \mathrm{e}+8$ & $9.829 \mathrm{e}+8$ & $0.00 \%$ & 399 \\
PABLB & $1.108 \mathrm{e}+9$ & $1.108 \mathrm{e}+9$ & $0.01 \%$ & 672 \\
AEJAL & $1.490 \mathrm{e}+9$ & $1.490 \mathrm{e}+9$ & $0.00 \%$ & 415 \\
09_H2 & $2.120 \mathrm{e}+9$ & $2.115 \mathrm{e}+9$ & $0.22 \%$ & 8642 \\
11_H2 & $2.479 \mathrm{e}+9$ & $2.477 \mathrm{e}+9$ & $0.07 \%$ & 9411 \\
10_H1 & $2.259 \mathrm{e}+9$ & $2.255 \mathrm{e}+9$ & $0.19 \%$ & 7267 \\
10_H2 & $2.529 \mathrm{e}+9$ & $2.526 \mathrm{e}+9$ & $0.12 \%$ & 10649 \\
11_H1 & $3.217 \mathrm{e}+9$ & $3.214 \mathrm{e}+9$ & $0.09 \%$ & 10075 \\
HKHKG & $3.427 \mathrm{e}+9$ & $3.427 \mathrm{e}+9$ & $0.00 \%$ & 4344 \\
10_FY & $4.835 \mathrm{e}+9$ & $4.807 \mathrm{e}+9$ & $0.59 \%$ & 28922 \\
\hline
\end{tabular}

Table 7.4: Results and performance of DW implementation. Instance is the name. $O b j_{D W}$ is the best found solution for algorithm, $L B_{D W}$ is the best found lower bound. Gap ${ }_{D W}$ is the resulting gap between upper and lower bound and $t_{D W}$ is the timed used in seconds.

\begin{tabular}{|l|c|c|c|}
\hline DualBoxSize & DTime & DObjective & Iterations \\
\hline 10 & $85.3 \%$ & $0.026 \%$ & 39 \\
50 & $34.0 \%$ & $0.061 \%$ & 26 \\
75 & $8.4 \%$ & $0.052 \%$ & 20 \\
100 & $15.9 \%$ & $0.056 \%$ & 20 \\
150 & $3.8 \%$ & $0.095 \%$ & 20 \\
1000 & $8.2 \%$ & $0.088 \%$ & 20 \\
\hline
\end{tabular}

Table 7.5: Parameter tuning for the Box step size used for Dual variable stabilization. $\Delta$ Time is the average percentage improvement in running time as compared with the fastest overall, taken over 5 medium and large problem instances. Likewise $\Delta$ Objective is the average percentage improvement in objective value. Iterations gives the average number of iterations to solve the root node.

\begin{tabular}{|l|c|c|c|}
\hline InitSubGap & DTime & Dobjective & Iterations \\
\hline 0.00001 & $10.8 \%$ & $0.078 \%$ & 19 \\
0.0001 & $11.9 \%$ & $0.064 \%$ & 19 \\
0.001 & $9.3 \%$ & $0.085 \%$ & 19 \\
0.01 & $10.3 \%$ & $0.054 \%$ & 19 \\
0.1 & $15.4 \%$ & $0.052 \%$ & 19 \\
0.5 & $30.2 \%$ & $0.081 \%$ & 21 \\
\hline
\end{tabular}

Table 7.6: Parameter tuning for the initial gap the subproblems are allowed to terminate with. $\Delta$ Time is the average percentage improvement in running time as compared with the fastest overall, taken over 5 medium and large problem instances. Likewise $\Delta$ Objective is the average percentage improvement in objective value. Iterations gives the average number of iterations to solve the root node. 


\begin{tabular}{|l|c|c|c|}
\hline GapIncrement & $\Delta$ Time & $\Delta$ Objective & Iterations \\
\hline 2 & $20.2 \%$ & $0.044 \%$ & 18 \\
5 & $6.4 \%$ & $0.088 \%$ & 17 \\
10 & $9.0 \%$ & $0.052 \%$ & 17 \\
25 & $6.1 \%$ & $0.041 \%$ & 17 \\
100 & $12.2 \%$ & $0.044 \%$ & 17 \\
\hline
\end{tabular}

Table 7.7: Parameter tuning for the factor the sub problem gap is reduced with. $\Delta$ Time is the average percentage improvement in running time as compared with the fastest overall, taken over 5 medium and large problem instances. Likewise $\Delta$ Objective is the average percentage improvement in objective value. Iterations gives the average number of iterations to solve the root node. 


\section{Part IV}

\section{Conclusion}





\section{Chapter 8}

\section{Conclusion}

Two overall research paths have been followed within liner shipping in this thesis: How to construct methods to assist with the design of liner shipping networks and how to model operational problems met in liner shipping. It has aimed at opening up research in the important area of liner shipping network design in a number of ways. By giving a thorough introduction to the domain, presenting a number of benchmark instances and proposing several models for liner shipping network design, which highlights important and not previously studied aspects of the problem. The mathematical models aid future research into LSNDP and the design of algorithms to solve significant instances of the problem. LINER-LIB 2012 data is already used by researchers from a number of countries around the world (Germany, Norway, Denmark, China, Singapore), for network design and other liner shipping projects. With the new research project, the Competitive Liner Shipping Network Design project ${ }^{1}$ an important foundation for research and industry collaboration is laid, which can be used by the world's liner shipping companies in the future, to optimize their networks. The project extends, among others, on the work of this thesis.

Operational liner shipping problems on bunker purchasing and disruption management, have been considered with good results, showing the breadth of research existing in liner shipping. From an industry point of view both operational models are applicable for implementation in actual decision support systems. These can help overcome some of the complex problems faced in liner shipping, showing that OR techniques can be applied to real liner shipping problems. This chapter highlights the main findings of the thesis, summarizing the conclusions of the works herein. It is followed by some thoughts on current trends in liner shipping network design research, and research in other liner shipping problems.

The paper in Chapter 2 highlights the potential for making cost effective and energy efficient liner shipping networks using OR. It is argued that a reason for lacking research has been, that access to domain knowledge and data is a barrier for researchers to approach the important liner shipping network design problem. The purpose of the benchmark suite and the paper is to provide easy access to the domain and the data sources of liner shipping for OR researchers in general. The liner shipping domain is analyzed and applied to network design and present a rich integer programming model based on services. It is proven that the liner shipping network design problem is strongly NP-hard. A benchmark suite of data instances to reflect the business structure of a global liner shipping network is presented. The design of the benchmark suite is discussed in relation to industry standards, business rules and mathematical programming. Computational results yielding the first best known solutions for 6 of the 7 benchmark instances is provided using a heuristic combining tabu search and heuristic column generation.

\footnotetext{
${ }^{1} \mathrm{~A}$ recently started research project at DTU Management, in cooperation with Maersk Line and funded by the Danish Maritime Fund.
} 
The paper in Chapter 3 develops a method to guide the optimal deployment of vessels. A single vessel round trip is considered by minimizing operational costs and flowing the best paying demand under commercially driven constraints. The Single Service Design Problem have been introduced and arc-flow and path-flow models are presented. A Branch-and-Cut-and-Price algorithm is proposed and implemented. The algorithm can solve instances of up to 25 ports to optimality - a very promising result as real-world vessel roundtrips seldom involve more than 20 ports.

Chapter 4 presents a new path based MIP model for the Liner Shipping Network Design Problem. The proposed model reduces problem size using a novel aggregation of the demands. A decomposition method enabling delayed column generation is presented. The subproblems have similar structure to Vehicle Routing Problems, which can be solved using dynamic programming. An algorithm has been implemented for this model, unfortunately with discouraging results due to the structure of the subproblem and the lack of proper dominance criteria in the labeling algorithm.

In Chapter 5 a novel compact formulation of the liner shipping network design problem is presented based on service flows. The formulation alleviates issues faced by arc flow formulations with regards to handling multiple calls to the same ports, butterfly ports, which previously has not been fully considered by liner shipping network design problem formulations. The method introduces service nodes, together with port nodes in a graph of the problem. Arcs from a port node to a service node represents whether a service is calling a port, and the demand load / unloads, at the port call. This representation allows any number of butterfly ports, which other models have not handled, while generating multiple interconnected services. The model is solved as a Mixed Integer Program. Results are presented for the two smallest instances of the benchmark suite LINER-LIB 2012 presented in Chapter 2

Chapter 6 investigates the huge impact of operational disruptions in liner shipping on costs and delayed cargo. The Vessel Schedule Recovery Problem evaluates a given disruption scenario and selects a set of recovery actions balancing the trade off between increased bunker consumption and the impact on cargo in the remaining network. It is proven that the Vessel Schedule Recovery Problem is $\mathcal{N} \mathcal{P}$-hard. The model is applied to four real life cases from Maersk Line and solutions are found in less than 5 seconds with results comparable or superior to those chosen by operations managers in real life. Cost savings of up to $58 \%$ may be achieved by the suggested solutions compared to realized recoveries of the real life cases.

Chapter 7 considers bunker purchasing for a liner shipping company. The cost for bunker fuel represents a major part of the daily running cost of liner shipping vessels. The vessels, sailing on a fixed roundtrip of ports, can lift bunker at these ports. The ports have differing and fluctuating prices. Contracts are often used to purchase bunker, ensuring supply and often at a discounted price. A contract can supply any vessel in a period and port, and is a shared resource between vessels, which must be distributed optimally to reduce overall costs. The Bunker Purchasing with Contracts Problem has been formulated as a mixed integer program, which has been Dantzig-Wolfe decomposed. To solve it, a column generation algorithm has been developed. The algorithm has been run on a series of real world instances with up to $500+$ vessels and $500+$ contracts, and provide near optimal solutions. A MIP model cannot solve these instances due to memory requirements.

\subsection{Contribution}

The contributions of this thesis' research papers are: 
- The liner shipping network design benchmark data set of LINER-LIB 2012. This detailed real life data set allows OR researchers to investigate the LSNDP and compare developed methods. A detailed domain description scopes liner shipping network design in terms of OR.

- A basic model for the LSNDP, which captures its core structures. An algorithm has been constructed and implemented, reporting the first results for the LINER-LIB 2012 instances.

- A path based model for a liner shipping problem is presented together with a novel aggregation scheme for the demands, which greatly reduces the number of demands that must be considered.

- A model and algorithm is developed for constructing a single liner shipping service, which can solve instances of real world size, of up to to 25 ports. While considering vessel capacity and demand path duration limits. The path durations limits are a key commercial factor in designing services to guarantee customer service levels.

- A novel service flow formulation for a liner shipping network design problem is presented, which allows for multiple interconnected services with any number of recurrent port calls to a port, a problem not solved by other liner shipping models. The implementation finds solutions for two of the LINER-LIB 2012 instances.

- A model and experimental results are presented for the Vessel Schedule Recovery Problem, which can be used for disruption management in liner shipping. Savings of up to $58 \%$ are reported compared with actual chosen recoveries.

- A model, algorithm and implementation considering the problem of purchasing bunkers for a fleet of scheduled vessels, while considering a number of bunker contracts, is presented. Managing this huge cost effectively seen over a fleet of vessels, can have significant economical impact.

\subsection{Trends in Liner Shipping Optimization Research}

Current trends for research in liner shipping network design and other optimization problems in liner shipping are discussed in this section.

\subsubsection{Liner Shipping Network Design}

Most of the investigated algorithms for liner shipping network design in this thesis are based on optimal methods. The outcome of these studies has been an elaborated understanding of LSNDP problems, their structure and their complexity. But it has also highlighted that optimal methods are unlikely to scale to the problems sizes of real world instances with hundreds of ports and vessels and thousands of demands. To tackle these large problems, a focused effort on developing heuristic methods, handling the complex landscape of operational and commercial constraints found in LSNDP problems, is needed. Such steps have already been taken in the works of Agarwal and Ergun [1, Alvarez [3, Wang et al. 11] and Chapter 2, but further work is needed to include important constraints as transit time limits, empty container repositioning and others. This could be a closer study of state of the art heuristic methods such as Pisinger and Røpke [8]. This approach could lead to good solutions for the largest instances of LINER-LIB 2012.

Should an optimal method be pursued for LSNDP, the method of Stålhane et al. [10] could be investigated, where a column generation method, generates new rows associated with new columns, but still achieves optimality. A decomposition of the service flow model of Plum et al. 9 could be based on this idea.

An even more challenging problem will be to develop methods that can improve on the networks of actual liner shipping network carriers. Due to the complexity of the networks, the uncertainty of 
the demand and the scale of the business, it is unlikely that such networks will ever be optimized using a blank sheet approach. When the best methods in the literature consider significant parts of the complexity of the problem, tools might be developed that can optimize on real liner shipping networks, in an incremental approach. E.g. by fixing large parts of the network design problem, while keeping the flow of demand open, a tool can optimize effectively on the small free part of the network. This has the advantage in an actual implementation phase, that network changes can be managed, using company practice for network design, and the size of the changes can be limited to what the organization can control and have faith in.

Two projects are currently following this path. A matheuristic was presented in Brouer and Desaulniers [6], which is run on the LINER-LIB 2012 instances. The column generation inspired local search method, flows the demand in a master problem and creates, destroys and modifies new services in a local search algorithm. The LINER-LIB 2012 was solved using an initial construction heuristic, the solution was then refined by the matheuristic. The results are comparable with those of Chapter 2. This method was further developed in Brouer [5 to take offset in an existing liner shipping network, resembling a global carriers network. The approach would then fixing parts of this network and optimize on the free part. The algorithm will find improved solutions in the local search neighborhood of this restricted network design problem. The results are encouraging and validated by the global carrier as being interesting, but still lacking constraints as transit time limits on demands.

A similar approach is being pursued in Wang et al. 11, where a heuristic network design algorithm takes offset in the existing network of a global liner shipping carrier. Parts of the network is fixed and new services are generated and evaluated for entry in the network. The method is reported to improve the network measured on key KPI's.

On a longer term a great challenge for the research in liner shipping network design, is how to face the complex world of competition and collaboration existing in liner shipping, as the number of alliances and vessel sharing agreements between competing carriers increase. This problem opens even greater depths of complexity. For the next years focusing on deterministic one carrier versions of the problem will be challenging, but further in the horizon, even greater tasks await.

Stochastic versions of the liner shipping network design problem could be considered. For instance the demand or parts of the costs could be viewed as uncertain, which would pose a considerable challenge.

This thesis will aid the quest for efficient heuristics in several ways. With suggestions on how to model and solve problem aspects as transit time limits, butterfly services, etc. Good heuristic often have an inspiration from optimal methods, guiding towards problem aspects that can be managed easily with efficient methods, but also highlighting which aspect are hard computational and burdened by degeneration and symmetry. A lesson, from this thesis could be that the combined solving of the flow problem and network design problem in one model, greatly impacts the scalability of the method. Splitting the flow and design problem in two phases as master and sub problems seems needed.

To evaluate the quality of a heuristic, it its powerful to have optimal solutions to compare against, to get bounds. For instance as done in Chapter 5 providing initial bounds on the heuristic solutions of Chapter 2

Heuristic methods show the way for solving large liner shipping network design problems, by fixing significant parts of the network, while flowing the demand freely and optimizing on the remaining free network.

The aim of Chapter 2, to unite the research in liner shipping network design by formulating a full model of the problem, is probably not reached yet. To illustrate, this thesis proposes a number of different models for the problem highlighting the need for alternate models. Furthermore the formulation of Chapter 2 has shortcomings, as being exponential and not including constraints as time limits. The quest for the ultimate liner shipping network design model is still open. 


\subsubsection{Other Liner Shipping Problems}

Interesting challenges of great practical relevance exist in other problems met in liner shipping. An initial draft of a project for a feeder network design problem, considering ports served first come, first serve is presented in Appendix Chapter 10. Other interesting research projects could extend on the fleet asset management approach in Alvarez et al. 4] investigated for bulk shipping. This could be extended to consider liner shipping. Little research has been done on liner shipping yield management, which could be interesting to investigate. As could the game theoretic approaches of Agarwal and Ergun 2 and their effect on important Vessel Sharing Agreements and carrier alliances. Eefsen and Cerup-Simonsen 7 . considers the effects of inventory costs of goods transported in containers, and the economic impact of speed reduction, an interesting approach that could be investigated further in liner shipping network design.

\subsection{Final Remarks}

This thesis has investigated methods for the liner shipping network design problem. The domain has been described in the terms of an OR professional. The LINER-LIB 2012 benchmark instances have been presented, and are already showing the first signs of opening the research field to new researchers. Four different network design models have been formulated and implemented, investigating important aspects of the problem as scalability, decomposition, butterfly ports and transit time on demands. These have provided results for the LINER-LIB 2012 instances. Two decision support methods for bunker purchasing with contracts and vessel schedule recovery, have been proposed, implemented and tested on real problems with promising results.

These works highlight the relevance and importance of developing optimization methods for planning and execution problems met in liner shipping. 


\section{Bibliography}

[1] Richa Agarwal and Ozlem Ergun. Ship scheduling and network design for cargo routing in liner shipping. Transportation Science, 42(2):175-196, 2008.

[2] Richa Agarwal and Ozlem Ergun. Mechanism design for a multicommodity flow game in service network alliances. Operations Research Letters, 36(5):520 - 524, 2008. doi: 10.1016/ j.orl.2008.04.007.

[3] J. Fernando Alvarez. Joint routing and deployment of a fleet of container vessels. Maritime Economics and Logistics, 11:186-208, 2009.

[4] J. Fernando Alvarez, Panagiotis Tsilingiris, Erna Engebrethsen, and Nikolaos M. P. Kakalis. Robust fleet sizing and deployment for industrial and independent bulk ocean shipping companies. INFOR, Special Issue on Maritime Transportation.

[5] Berit D. Brouer. Liner Service Network Design. PhD thesis, The Technical University of Denmark, Management Engineering, 2012.

[6] Berit D. Brouer and Guy Desaulniers. A matheuristic for the liner shipping network design problem. In Proceedings of the second International Conference on Logistics and Maritime Systems, Bremen, 2012.

[7] Thomas Eefsen and Bo Cerup-Simonsen. Speed carbon emissions and supply chain in container shipping. In Proceedings of the Internatioanl Association of Maritime Economists Conference, Lisbon, July 2010.

[8] David Pisinger and Stefan Røpke. Large neighborhood search. In Handbook of metaheuristics, pages 399-419. Springer, 2010.

[9] Christian E. M. Plum, David Pisinger, and Mikkel M. Sigurd. A service flow model for the liner shipping network design problem. Submitted for special issue of European Journal of Operational Research on Maritime Logistics, 2012.

[10] Magnus Stålhane, Henrik Andersson, Marielle Christiansen, Jean-François Cordeau, and Guy Desaulniers. A branch-price-and-cut method for a ship routing and scheduling problem with split loads. Computers $\mathcal{E}$ Operations Research, 2012.

[11] Shuaian Wang, Zhiyuan Liu, and Qiang Meng. Systematic network design for liner shipping services. To appear in Transportation Research Record. 